\title{
Combustion synthesis and structural characterization of Li-Ti mixed nanoferrites
}

\author{
R M SANGSHETTI ${ }^{\dagger}$, V A HIREMATH ${ }^{\dagger \dagger}$ and V M JALI* \\ Department of Physics, Gulbarga University, Gulbarga 585 106, India \\ ${ }^{\dagger}$ Department of Physics, ${ }^{\dagger \dagger}$ Department of Chemistry, P.D.A. College of Engineering, Gulbarga 585 102, India
}

MS received 26 October 2010; revised 9 March 2011

\begin{abstract}
Polycrystalline samples of the mixed nanoferrites, $\mathrm{Li}_{0.5+0.5 x} \mathrm{Ti}_{x} \mathrm{Fe}_{2.5-1.5 x} \mathrm{O}_{4}(0.02 \leq x \leq 0 \cdot 1)$, were prepared by combustion method at lower temperatures compared to the conventional high temperature sintering for the first time at low temperatures, using PEG which acts as a new fuel and oxidant. XRD patterns reveal a singlephase cubic spinel structure. The as synthesized $\mathrm{Li}$-Ti ferrites are in nanocrystalline phase. The crystallite size was found to be in the range 16-27 $\mathrm{nm}$. SEM images reveal rod-like morphology in all the samples with a discontinuous grain growth. The B-H loops have been traced using VSM technique, for all the compositions, at room temperature and the hysteresis parameters are calculated. Saturation magnetization decreases with increase in Ti content due to the fact that the $\mathrm{Ti}^{4+}$ ion, which is a non-magnetic ion, replaces a magnetic $\mathrm{Fe}^{3+}$ ion. The hysteresis loops show clear saturation at an applied field of $\pm 10 \mathrm{kOe}$ and the loops are highly symmetric in nature. The cation distribution is known indirectly by using saturation magnetization values.
\end{abstract}

Keywords. Li-Ti mixed ferrites; combustion synthesis; hysteresis.

\section{Introduction}

Lithium ferrites are low cost materials, which are generally found useful in microwave-device and memory-core applications (Baba and Argentina 1975; Dionne 1975; Kulshreshtha and Ritter 1985; Bermejo et al 1994). The modifications in the properties of ferrites due to substitution of different ions, those dependent upon the nature and number of substituted ions, have been studied by various workers. Several studies (Watanabe et al 1981; Gill and Puri 1985; Reddy et al 1988) have been reported with additions of divalent, trivalent and tetravalent ions to monitor various parameters, depending on the desired applications of ferrites. Non-magnetic substitution in ferrites alter the inter and intra-sub lattice exchange interactions between the magnetic ions giving rise to a variety of interesting magnetic properties (Neel 1951). Substitution of non-magnetic ions weakens the exchange interaction and can lead to the formation of paramagnetic clusters (Gilleo 1960), localized canting (Nicolas et al 1973), etc altering the net magnetic moment. A study of magnetization is useful in understanding the arrangement of spins and the distribution of magnetic cations in the sublattice. On the other hand, mixed lithium ferrite

\footnotetext{
*Author for correspondence (vmjali@gmail.com)
}

has been used as a promising substitute for $\mathrm{NiCuZn}$ ferrites for advanced planar ferrite devices, such as multilayer chip inductors (MLCIs), because of their low sintering temperature, high Curie temperature and excellent electromagnetic properties at high frequency (Zhou et al 1998).

It is well known that low temperature sintering of ferrites can be achieved by using active ultrafine powders synthesized via a wet chemical method. Several chemical processing techniques, such as hydrothermal, sol-gel, coprecipitation and combustion synthesis have been investigated to prepare ultrafine ferrite powders (Fujimoto 1994; Dias and Buono 1997; Ravindranathan and Patil 1987; Kumar et al 1996; Suresh and Patil 1992; Cho et al 1999). Among these techniques, combustion synthesis has been proved to be a simple and economic way to prepare nanoscale powders (Chakrabarti and Maiti 1997; Schafer et al 1997; Yue et al 1999). In this technique, a thermally induced redox reaction takes place and the reaction is exothermic. The energy from the exothermic reaction between oxidant and reductant can be high enough to form a desirable phase within a very short time. High temperature synthesis of Li ferrites involves volatilization of lithium and oxygen at a temperature above $1000^{\circ} \mathrm{C}$, which affects the magnetic properties (White and Patton 1978). In view of this, to reduce the loss of lithium content, an attempt has been made to synthesize the Li-Ti mixed ferrites employing combustion synthesis, at different compositions, at a reduced temperature of sintering compared to the conventional high 
temperature synthesis routes reported in literature (Reddy et al 1988; Wafik et al 1993).

\section{Experimental}

Li-Ti mixed ferrites with the compositional formula, $\mathrm{Li}_{0.5+0.5 x} \mathrm{Ti}_{x} \mathrm{Fe}_{2.5-1.5 x} \mathrm{O}_{4}(0.02 \leq x \leq 0.1)$, were prepared by combustion method using high-purity analytic reagents $\mathrm{LiCO}_{3}, \mathrm{Fe}_{2} \mathrm{O}_{3}, \mathrm{TiO}_{2}$ and polyethylene glycol (PEG). PEG (molecular weight, 6000) acts as a new fuel for combustion synthesis. All the initial reagents were mixed with PEG in a weight ratio of 1:2 and ground well in a pestle and mortar. The resulting mixture was placed in an alumina crucible and sintered at a temperature of $350^{\circ} \mathrm{C}$ for $3 \mathrm{~h}$. The reacted powders were ball milled with distilled water as wetting agent for $5 \mathrm{~h}$ and dried. Phase confirmation and structure determination were done using X-ray diffraction (XRD, Philips PW 1051). The structure and lattice parameter values were obtained using the 'POWDER X' software. Scanning electron microscope was used to study the powder surface morphology (SEM, Leica - 440 Cambridge Stereoscan). The average crystallite size was calculated by the well known Scherrer's equation. Vibrating Sample Magnetometer (VSM, Quantum model - VSM 6000) at an applied field of $\pm 10 \mathrm{kOe}$ was used for study of magnetization. Small amounts of the sample $(0.05 \mathrm{~g})$ were placed in a glass ampoule of $1 \mathrm{~mm}$ inner diameter and mounted in VSM. The magnetization variation was studied as a function of magnetic field at room temperature for all compositions and hysteresis parameters were calculated.

\section{Results and discussion}

\subsection{Synthesis}

Combustion synthesis, which is a quick and straightforward preparation process to produce homogeneous, crystalline and unagglomerated multi component oxide ceramic powders without the intermediate decomposition and/or calcining steps, was used to prepare $\mathrm{Li}-\mathrm{Ti}$ mixed nanoferrites. These samples were successfully prepared for the first time at lower temperatures compared to conventional high temperature sintering, by combustion method using PEG which acts as a new fuel and oxidant.

\subsection{Structural characterization}

Figure 1 shows the comparison of XRD patterns of $\mathrm{Li}_{0.5+0.5 x} \mathrm{Ti}_{x} \mathrm{Fe}_{2.5-1.5 x} \mathrm{O}_{4}(0.02 \leq x \leq 0.1)$ samples in the $20-70^{\circ} 2 \theta$ range. The main reflection planes appear in all patterns. XRD analysis shows the single-phase cubic spinel structure and was confirmed by indexing all major peaks with 'POWDER X' software and lattice constant for all compositions was found to be $0.833 \mathrm{~nm}$ which is consistent with

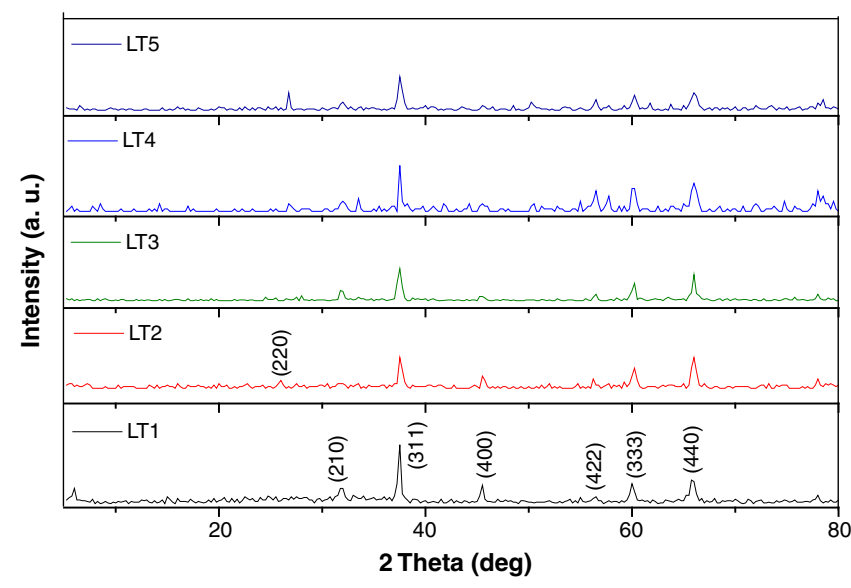

Figure 1. Comparison of XRD pattern of LT1 to LT5 samples at room temperature.

those reported by Yousif et al (1994). The theoretical lattice constants $\left(a_{\mathrm{th}}\right)$ were estimated from the ionic radii of the A sites $\left(r_{\mathrm{A}}\right), \mathrm{B}$ sites $\left(r_{\mathrm{B}}\right)$ and the radius of oxygen ion $\left(r_{\mathrm{O}}\right)$ (Mazen et al 1993) using

$$
a_{\mathrm{th}}=(8 / 3 \sqrt{ } 3)\left[\left(r_{\mathrm{A}}+r_{\mathrm{O}}\right)+\sqrt{ } 3\left(r_{\mathrm{A}}+r_{\mathrm{O}}\right)\right] .
$$

These theoretical lattice parameter values were found to be $0.833 \mathrm{~nm}$. These values are consistent with those reported by Yousif et al (1994). The crystallite sizes of the synthesized powders were determined using Scherrer's equation

$$
D=\frac{0 \cdot 9 \lambda}{\beta \cos \theta},
$$

where $D$ is particle size, $\lambda$ the wavelength of $\mathrm{CuK}_{\alpha}, \beta$ the FWHM and $\theta$ the diffraction angle.

Table 1 shows the average crystallite size, saturation magnetization and coercivity values. The synthesized powders have nanocrystalline particles with crystallite size, $16-27 \mathrm{~nm}$.

\subsection{Microstructure analysis}

Figure 2 shows SEM images at a magnification of $\times 20 \mathrm{~K}$ of LT1 and LT4 samples. Rod-like morphology was observed in all the samples with discontinuous grain growth. No other different morphological grains were observed indicating that no second phase was present.

\section{$3.4 V S M$}

The primary function of $\mathrm{Ti}$ is to lower saturation magnetization $\left(M_{\mathrm{s}}\right)$ value. As in all ferrites, the magnetization may be reduced by the substitution of various non-magnetic 
Table 1. Crystallite size, saturation magnetization and coercivity values.

\begin{tabular}{lcccc}
\hline Ferrite & Sample code & $\begin{array}{c}\text { Crystallite } \\
\text { size }(\mathrm{nm})\end{array}$ & $\begin{array}{c}\text { Saturation magnetization, } \\
M_{\mathrm{S}}(\mathrm{emu} / \mathrm{g})\end{array}$ & $\begin{array}{c}\text { Coercivity, } \\
H_{\mathrm{c}}(\mathrm{Oe})\end{array}$ \\
\hline $\mathrm{Li}_{0.5+0.5 x} \mathrm{Ti}_{x} \mathrm{Fe}_{2 \cdot 5-1.5 x} \mathrm{O}_{4}(x=0 \cdot 02)$ & $\mathrm{LT} 1$ & $19 \cdot 59$ & $57 \cdot 05$ & 146 \\
$\mathrm{Li}_{0.5+0.5 x} \mathrm{Ti}_{x} \mathrm{Fe}_{2 \cdot 5-1.5 x} \mathrm{O}_{4}(x=0 \cdot 04)$ & $\mathrm{LT} 2$ & 22.08 & 56.40 & 144 \\
$\mathrm{Li}_{0.5+0.5 x} \mathrm{Ti}_{x} \mathrm{Fe}_{2 \cdot 5-1.5 x} \mathrm{O}_{4}(x=0 \cdot 06)$ & LT3 & $16 \cdot 13$ & $55 \cdot 00$ & 180 \\
$\mathrm{Li}_{0.5+0.5 x} \mathrm{Ti}_{x} \mathrm{Fe}_{2 \cdot 5-1.5 x} \mathrm{O}_{4}(x=0 \cdot 08)$ & LT4 & 26.88 & $54 \cdot 80$ & 143 \\
$\mathrm{Li}_{0.5+0.5 x} \mathrm{Ti}_{x} \mathrm{Fe}_{2 \cdot 5-1.5 x} \mathrm{O}_{4}(x=0 \cdot 10)$ & LT5 & 19.06 & 52.96 & 137 \\
\hline
\end{tabular}

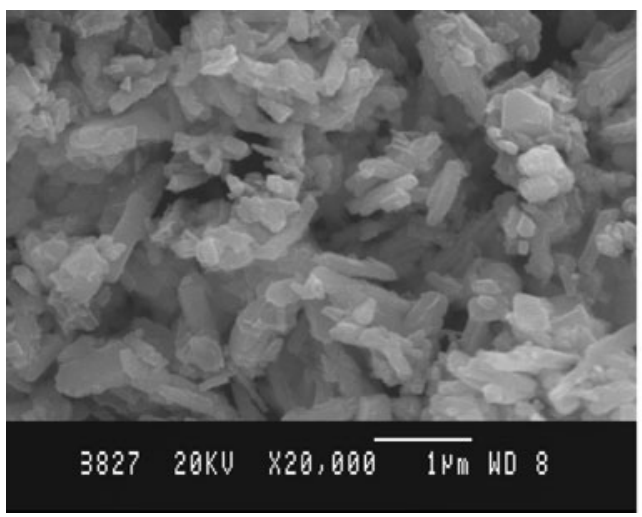

(a) LT1

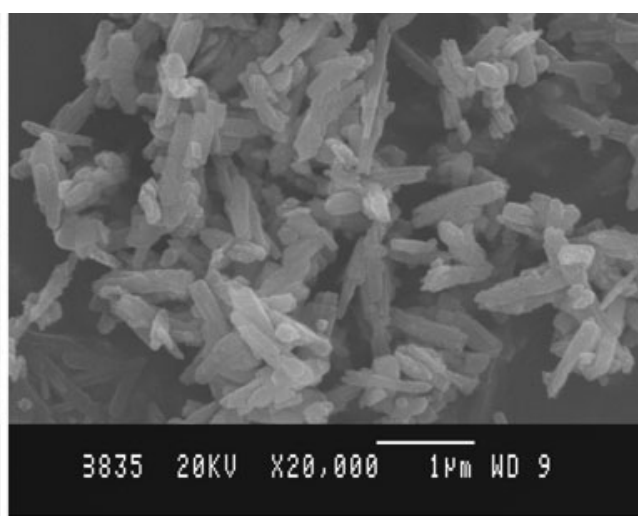

(b) LT4

Figure 2. SEM images of LT1 and LT4 samples at room temperature.
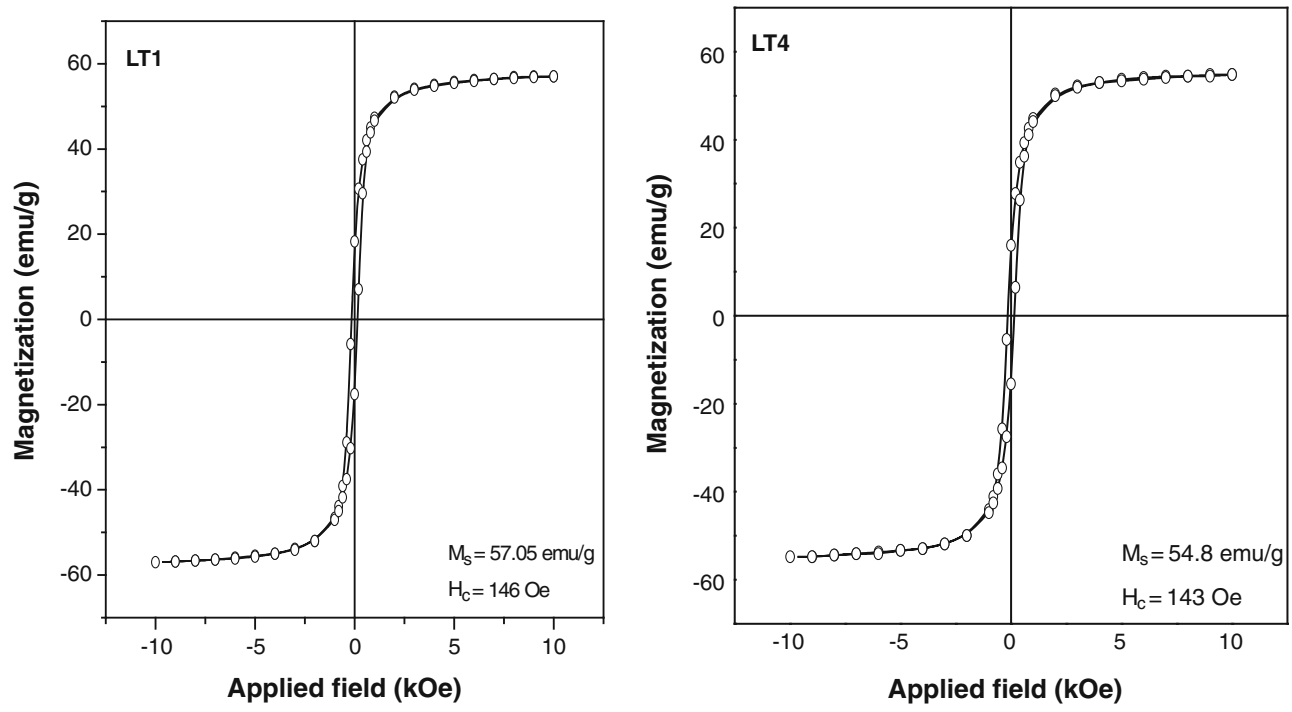

Figure 3. VSM curves for LT1 and LT4 samples at room temperature. 
ions for Fe ions in the octahedral sublattice (Baba et al 1972). Figure 3 shows VSM curves for LT1 and LT4 samples, at room temperature. Magnetization values were obtained using vibrating sample magnetometer (VSM) and are shown in table 1 for different Ti contents. $M_{\mathrm{s}}$ value decreases with increase in Ti content due to the fact that the $\mathrm{Ti}^{4+}$ ion, which is a non-magnetic ion, replaces a magnetic $\mathrm{Fe}^{3+}$ ion. The variation of saturation magnetization with dopant concentration is shown in figure 4 . It can be seen from the figure that the values of saturation magnetization decreases with increase in Ti concentration. The coercivity value decreases with increase in Ti-content except in LT3 sample. We cannot establish clearly the inverse nature of coercivity with crystallite size. The hysteresis loops show clear saturation at an applied field of $\pm 10 \mathrm{kOe}$. The loops are highly symmetric

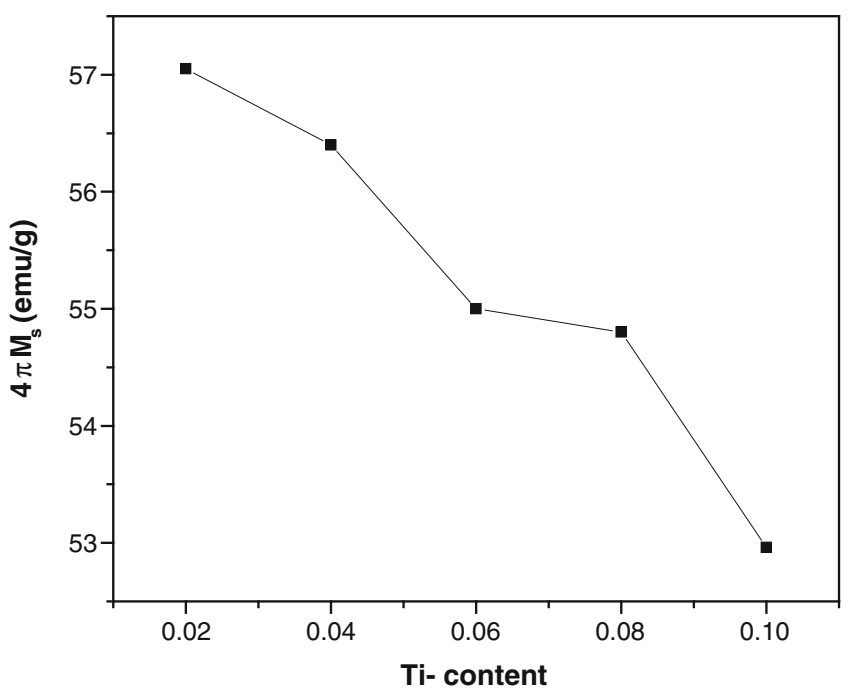

Figure 4. Variation of saturation magnetization with Ti-content.

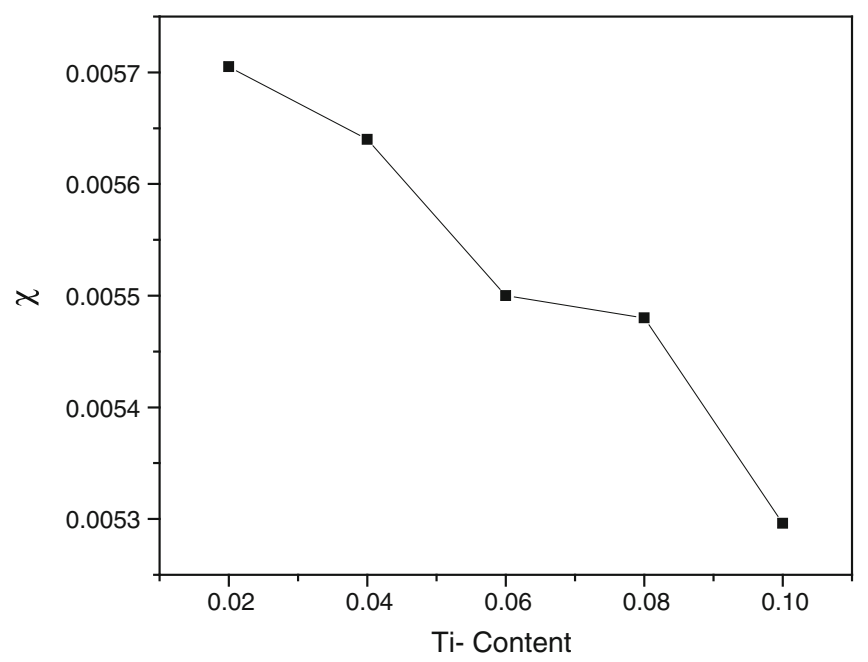

Figure 5. Variation of magnetic susceptibility with Ti-content. in nature. Magnetic susceptibility $(M / H)$ decreases with increase in Ti-content and is as shown in figure 5.

\section{Conclusions}

Li-Ti mixed ferrites were synthesized at different compositions at a reduced temperature of sintering compared to the conventional high temperature synthesis. XRD pattern reveals a single-phase cubic spinel structure. The results revealed that the nanocrystalline $\mathrm{Li}-\mathrm{Ti}$ ferrite powders directly formed after combustion and that the products' properties showed good reproducibility. SEM images reveal rod-like morphology with discontinuous grain growth. The saturation magnetization value decreases with increase in Ti content due to the fact that the $\mathrm{Ti}^{4+}$ ion, which is nonmagnetic, replaces a magnetic $\mathrm{Fe}^{3+}$ ion. For ferrite application in recording media, low coercivity is required while maintaining high saturation magnetization. In the present case, however, coercivity has been lowered due to Ti substitution without maintaining high saturation magnetization. As $\mathrm{Ti}^{4+}$ is diamagnetic in nature, this observation is expected. As it is well known, squareness of hysteresis loop has attracted considerable attention. The present study also presents such squareness of the hysteresis loops. Following the work by Argentina and Baba (1974), when titanium ions are introduced in lithium ferrite, as the saturation magnetization value is found to decrease with increasing Ti content, it can be concluded that these Ti dopants may occupy the octahedral sites replacing $\mathrm{Fe}^{3+}$ ions.

\section{References}

Argentina G M and Baba P D 1974 IEEE Trans. Micr. Theory Tech. MTT-22 652

Baba P D and Argentina G M 1975 IEEE Trans. Microwave Theory Tech. 22654

Baba P D, Argentina G M, Courtney W E, Dionne G F and Temme D H 1972 IEEE Trans. Magn. MAG-8 83

Bermejo E, Chassing J, Bizot D and Quarton M 1994 Mater. Sci. Eng. B22 73

Chakrabarti N and Maiti H L 1997 Mater. Lett. 30169

Cho Y S, Burdick V L and Amarakoon V R W 1999 J. Am. Ceram. Soc. 821416

Dias A and Buono V T 1997 J. Mater. Res. 123278

Dionne G F 1975 J. Appl. Phys. 453621

Fujimoto M 1994 J. Am. Ceram. Soc. 773065

Gill N K and Puri R K 1985 J. Mater. Sci. Lett. 4396

Gilleo M A 1960 J. Phys. Chem. Solids 1333

Kulshreshtha S K and Ritter G 1985 J. Mater. Sci. 203926

Kumar P S A, Shrotri J J and Kulkarni S D 1996 Mater. Lett. 27293

Mazen S A, Abdalla M H, Nakhla R I, Zaki H M and Metawe F 1993 Mater. Chem. Phys. 3435

Neel L 1951 J. Phys. Radium 12160

Nicolas J, Lagrange A, Sroussi R and Inglebert R L 1973 IEEE Trans. Mag. MAG-9 546 
Ravindranathan P and Patil K C 1987 Am. Ceram. Soc. Bull. 66688 Reddy P V, Reddy M B, Muley V N and Ramana Y V 1988 J. Mater. Sci. Lett. 71243

Schafer J, Sigmand W, Roy S and Aldinger F 1997 J. Mater. Res. 122518

Suresh K and Patil K C 1992 J. Solid State Chem. 9912

Watanabe A, Yamamura H, Moriyoshi Y and Shirasaki S 1981 Ferrites, proc. int. conf. Tokyo, Japan (eds) H Watanabe et al (Tokyo: Centre for Academic Publications) p. 170
Wafik A H, Mazen S A and Mansour S F 1993 J. Phys. D: Appl. Phys. 262010

White G O and Patton C E 1978 J. Magn. Magn. Mater. 9299

Yousif A A, Elzain M E, Mazen S A, Sutherland H H, Abdalla M H and Masour S F 1994 J. Phys.: Condens. Matter 65717

Yue Z, Li L, Zhou J, Zhang H and Gui Z 1999 Mater. Sci. Eng. B64 68

Zhou J, Liang B, Zhang H, Yun Zi, Gui Z and Li L 1998 High Tech. Lett. 839 